\title{
Research and practice of kinetic energy system risk control technology in tobacco enterprises
}

\author{
Wang Husong, Ni Zhenyu, Jin Wei, Huang Qizhong, Xiao Guihui*, Le Yuannan, Liu Jianmin, Hu Xiaohu, Chen Lifeng, \\ Fang Biao \\ Ningbo Cigarette Factory, Zhejiang China Tobacco Industry, Jiapu West Road No.2001, Fenghua Economic Development Zone, Ningbo \\ city, Zhejiang Province, China,postcode:315504
}

\begin{abstract}
The purpose of this paper is to provide a data-oriented risk prevention and control system for seven major kinetic energy supply systems (power distribution, dust removal, moisture removal and odor removal, air pressure, vacuum, process soft water and sewage station water manufacturing, boiler steam system, air conditioning). The traditional risk control means should be improved through systematic process and information technology, and the risk management of operation control should be gradually transformed from event-driven to data-driven, so as to strengthen the comprehensive warning and prevention and control of operational risks. The management mode of integrated management and control is "clear hierarchy, effective air separation, effective control in place, quick early warning and simple handling". Water, electricity, gas and steam of the power system are realized as part of the internal quality and process control of the cigarette brand, and all the control is pushed to the power supply terminal.
\end{abstract}

\section{INTRODUCTION}

According to the characteristics of the cigarette factories supply long-distance conveying, the stability of the power supply of energy is particularly important, long-distance conveying magnify kinetic energy supply instability, and unstable energy supply will directly affect the tobacco moisture in the manufacturing process, flow, temperature, or even indirectly affect the quality of cigarette products, especially the flavour and taste.Therefore, according to the requirement of high quality development put forward the new idea of power control: power seven related to production system (dust moisture removal, steam boiler, air compressor, high vacuum, the craft is cold water, and air conditioning) is an integral part of "cigarettes factory process control", dynamic services cannot be limited on the timely supply of energy, is to practise the service in the silk, the roll bag production line kinetic energy interface terminal.We need to study and practice the innovation of kinetic energy system risk control process in tobacco enterprises.

\section{RESEARCH AND PRACTICE ON THE ESTABLISHMENT OF RISK CONTROL SYSTEM}

\subsection{Creatively establish the technical framework for risk point management and control of the seven major kinetic energy supply systems}

The risk point management and control function adopts micro-service architecture, relies on advanced technologies such as streaming computing and big data, and integrates MES, energy management system, centralized control system, data center and other information resources on the basis of existing business processes and information implementation experience, so as to realize the whole-process management of "collection, early warning, analysis and response". In the production of perception, energy prediction, equipment control, balance between supply and demand, production security, etc, to realize information sharing, be based on production driven "precise kinetic energy supply, power control integration, scientific decision analysis", fully coordinated kinetic energy supply and demand of resources, gradually establish a power horizontal collaboration, vertical well versed in management, information connectivity, high share of resources integration service mechanism.

Through system integration, the risk point management and control system realizes the integration with MES system's production plan, work order information, process related information, products and other basic data information. Through the low-level development, the integration of centralized control system, OPC data, real-time database data, energy management system data and external meteorological information is realized, so as to provide comprehensive and complete data for the realization of unified monitoring, prediction, early warning and optimization of the platform.

As shown in Figure 1, the architecture of the system can be divided into data source, data processing layer, data storage layer, analysis and optimization layer, data service

* Corresponding author: 2535879510@qq.com 
layer and application presentation layer from the perspective of data flow processing

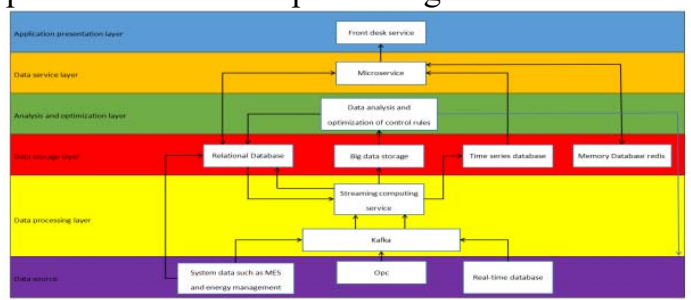

Figure. 1. System architecture diagram

The data sources for risk point control of power system come from many aspects, such as information of MES system and energy management system, external data such as weather service, as well as data acquisition information obtained from OPC server and sampling information extracted from real-time database.

The data of different information sources, according to a different approach, some data such as weather information, such as the repair order status information save directly to the relational database, the data need to processing, especially the flow calculation of data is sent to Kafka, after data processing, part of the data such as the alarm data to oracle, in history of monitoring information query data to influxdb temporal database, used in realtime monitoring of data sent to Kafka.

The data storage layer mainly includes relational database Oracle, big data storage Hadoop, time series database influxDB, and memory database RedIS. Oracle database mainly stores system related basic information, configuration information, model parameters, etc. Hadoop is used to store a large amount of processed data for analysis modeling and influxDB for historical query data; Redis to cache data that needs to be read and written repeatedly, such as warning rules.

The analysis and optimization layer mainly matches the specifications of equipment monitoring data and continuously optimizes them, and stores the optimized parameters in the risk point control standards.

The data service layer mainly provides data for microservices deployed in this layer. Micro-services obtain relevant data from the data storage layer and provide data services for application presentation and information push.

The application layer provides display services for front-end PC and mobile terminals, etc.

As shown in Figure 2, the system includes 6 servers, among which 2 serve as application servers to deploy microservice framework and application services. 2 as data processing and optimization modeling servers for data flow calculation and data analysis modeling; Two servers serve as data storage service groups, including one deployed Oracle relational database, one deployed XDB timing data, and so on.

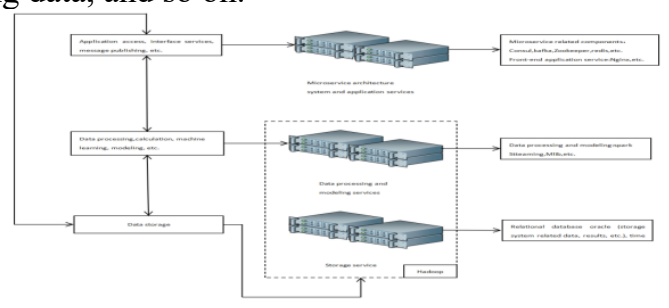

Figure. 2. System deployment diagram

Microservice architecture and application services mainly require response and processing performance. Therefore, clustering is recommended to improve the concurrency of the system and effectively balance the load

Data processing and analysis service requires realtime calculation and monitoring of data from OPC, timing database and related system interface, and there are a large number of data processing and analysis jobs, so it is recommended to deploy the cluster.

Because it involves big data computing and machine learning, you need to make sure there is a lot of space and can be expanded.

Data storage service that stores data analysis and processing results, modeling information, etc., both from relational database Oracle and from real-time computation and processing results (influxDB timing database); In addition, hadoop is adopted to store massive information to ensure sufficient storage space, and the space can be continuously expanded with the continuous accumulation of system operation and data.

Risk point control covers the whole kinetic energy system, including real-time flow design of boiler steam system, vacuum, air pressure system, air conditioning and refrigeration system, dust removal and moisture removal system, process cold water, power distribution system, sewage treatment system, etc. Through real-time monitoring of key indicators of kinetic energy operation, the system can predict and warn risk points, and push the results to relevant personnel through mobile phones in real time, so as to achieve comprehensive control of kinetic energy management. below.

The risk point processing process is shown in Figure 3

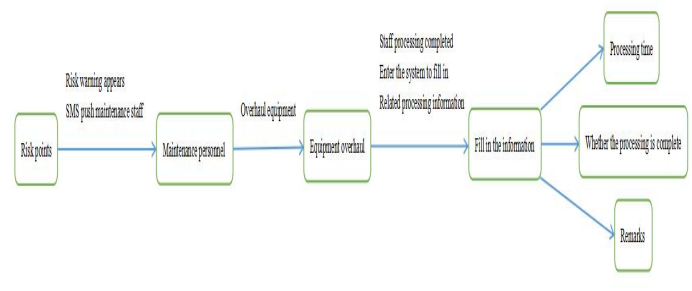

Figure. 3. Flow chart of risk points

According to the risk point detection standard, the system automatically detects the key risk point operation parameters of each kinetic energy system. When the operation index exceeds the control standard, the system will immediately push it to the operating team, relevant maintenance team and management personnel. After the operation team and maintenance personnel take on-site inspection, maintenance and other treatment measures, they will feed back the treatment process and measures to the system, so as to continuously provide data accumulation for the subsequent optimization of control standards and knowledge base.

\subsection{Establishment of risk point control data service platform}

In order to monitor, analyze and control the operation status of key nodes in the whole process of kinetic energy 
supply, this project designs a set of unified data aggregation and fusion architecture, establishes the core data support platform, and supports the operation of various business functions. The data processing process of the data service platform includes data acquisition, data management, data service and data application.

1) Data acquisition-Dynamic acquisition technology is used to gather real-time data of MES, energy management, power centralized control, equipment management and other systems and integrate historical data.

2) Data processing - To clean, convert, collect, organize, standardize and monitor the collected data to provide basic data meeting the requirements for data analysis;

3) Data modeling - The prediction, correlation analysis, anomaly detection and equipment control strategy of monitoring data under the background of kinetic energy big data were studied, and the data analysis model was established.

4) Data services-Design and implement data services based on the kinetic Internet of Things, and provide users and business systems with services or interfaces such as query, analysis, report, statistics and display; The upper data application makes authorized call to the data service and interface to support the operation of various business systems.

Based on the control requirements of power equipment, two modes, batch processing and flow calculation, are adopted for data on the platform to process data in different scenes respectively, so as to improve the processing capacity and layer expansion capacity of the system in the face of massive data.

\subsection{Key Technologies}

The integrated platform of intelligent power risk management and control is the implementation of the CPS system in the field of kinetic energy management and control in the tobacco industry. The platform adopts the framework system of micro-service and realizes efficient analysis, in-depth mining and application of massive data through flow computing.

1) Microservice framework

In recent years, with the rise and development of cloud computing, container virtualization, and DevOps integrating development, testing, deployment, and operation, microservice architecture has gradually become the mainstream technology. The basic idea is to divide the traditional single application into a series of software service units that can be independently designed, developed, deployed, operated and maintained according to the business functions, and the services cooperate with each other to realize the ultimate value. Microservices can be seen as a concrete implementation of a ServiceOriented Architecture (SOA), but they have advantages over Web services, such as decentralized management and lightweight container deployment, monitoring and early warning and multiple highly available fault tolerance strategies, and independent and free development of Service data.

The microservice architecture model has many advantages:

Componentization: Microservices can be thought of as components. The biggest difference from the traditional component approach is that the traditional component will apply modularity and build relatively independent units for it. Separate or extract common parts to build Shared libraries and achieve the effect of decoupling and reuse. Microservice architecture, on the other hand, decomposed the system into multiple services in a componentized way, with relatively independent and loosely coupled services. The change of a single function only requires the reconstruction and deployment of corresponding services.

Standalone deployment: Since microservices have separate running processes, each microservice can also be deployed independently. When a microservice changes, there is no need to compile and deploy the entire application, greatly reducing the application delivery cycle. The microservice architecture pattern makes continuous deployment possible.

Reducing complexity: try development status.Microservice architecture makes the complexity controllable and avoids unnecessary silos of data by decommissioning monolithic applications into multiple service methods. Under the condition of unchanged functions, the application is decomposed into multiple manageable services. Through the micro-service architecture pattern, the complex functions are presented in a modular way, providing modular solutions to the functions that are difficult to be realized by the single application coding method, and making the single service easier to develop and maintain

Technology diversification: The traditional development model is to use the same technology to build the entire application. Microservice architecture emphasizes a decentralized software organization architecture, enabling each service to independently choose the appropriate technology type and provide interface services according to its own service requirements and indus

Reduce the maintenance risk: Maintaining a large and complex single architecture system requires a lot of work, which is prone to lead to the whole system, so the risk is very high. In contrast, in the microservice architecture mode, when a component fails, there will be no defects such as in-process diffusion in the system of single block architecture, and the faults will be isolated in a single service, thus reducing the risk of maintenance and redevelopment.

2) Flow calculation

Streaming computing is a concept relative to batch computing, which refers to real-time calculation of incoming data stream in memory, while batch computing refers to centralized calculation of stored static data. Therefore, streaming computing has the characteristics of low delay, high throughput and continuous operation. In recent years, due to the high efficiency of distributed computing and real-time characteristics of data processing, streaming computing technology has been widely used in 
many fields such as finance, Internet and Internet of Things, such as real-time video analysis and real-time warning of traffic flow. Common streaming computing frameworks include Storm and SparkStreaming, etc. In this project, after the data is acquired through the data acquisition framework, the flow calculation can calculate the values of all kinds of monitoring indicators in a certain window in real time (for example, the steam flow and energy consumption within 5 minutes, etc.). If the deviation between the monitored index value and the historical or preset value exceeds the threshold value, automatic warning will be given.

\section{Case analysis and research on risk early-warning process control}

With the continuous advancement of intelligent factory, higher requirements have been put forward for equipment maintenance, and the original manual detection method has been unable to meet the requirements of intelligent factories at the present stage. Only the real-time monitoring of the equipment and the predictive maintenance and preventive maintenance according to the monitoring data of the equipment can improve the risk control ability of the system to meet the control requirements of modern factories.

The existing dust removal, moisture drainage, odor removal equipment related maintenance work are manual equipment of the indicators of regular testing and regular maintenance of the equipment. Daily maintenance mainly took the form of point inspection, the dust, moisture removal equipment of motor shaft temperature monitoring and equipment overall PaoMaoDiLou phenomenon does exist, in addition to the daily maintenance, and there are periodic equipment maintenance, equipment maintenance time is according to the time period specified in the manual system, artificial search system manual to arrange the maintenance plan. This kind of maintenance has certain risks. First, there are errors in manual detection. Secondly, manual detection cannot guarantee that the realtime equipment state meets the standard. Third, part of the data is not suitable for manual inspection, resulting in incomplete equipment detection; Fourth, due to the difference in the time of regular maintenance of each part of each equipment, the manual inquiry and rearrangement plan is too complicated and prone to omission.

1) Real-time push of risk warning information

According to the collected data and equipment maintenance experience, set the risk warning value. When the corresponding data of the equipment exceeds the risk warning value, contact the relevant person in charge through SMS.

2) Real-time push of equipment maintenance information

According to the periodic maintenance benchmark and real-time data of the equipment of dust removal, moisture removal and odor removal system, when the equipment arrives at the maintenance cycle or the real-time data indicates that the equipment needs maintenance, remind the relevant person in charge to arrange equipment maintenance through SMS.
3) Establishment of equipment risk point database

For the discovered risk points, a risk point database is specially established, in which the occurrence time, occurrence point location, the specific situation of the risk, the solution and the effect after processing are recorded.

The following Figure 4 is the system flow chart, which briefly explains the specific implementation steps of the data-oriented risk prevention and control method for dust removal, moisture removal and odor removal equipment.

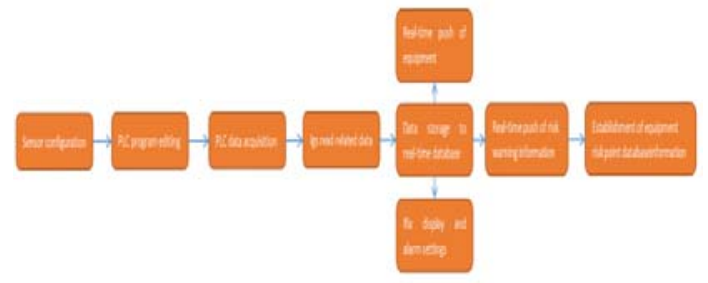

Figure. 4. System flow chart

The following is a brief introduction to the specific implementation.

1) Sensor configuration

As shown in Figure 5, install the additional sensors, such as SMT platinum thermal resistance MIK-PT100 (US Control) to monitor the temperature of the fan bearing, and complete the wiring connection with the PLC.

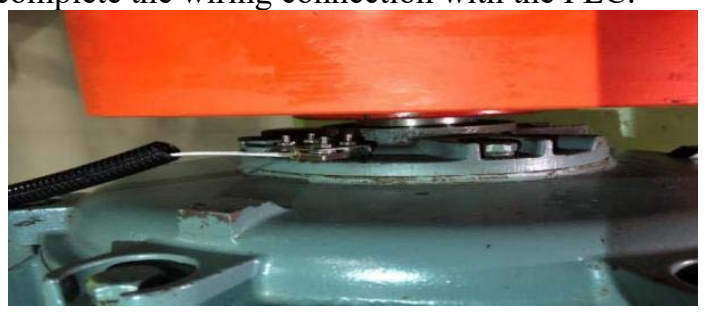

Figure. 5. Effect of sensor installation

\section{2) PLC program editing}

Program the PLC corresponding to the added sensor, such as the wafer platinum thermal resistance MIK-PT100 (US Control) for fan bearing temperature monitoring.

3) IGS reads relevant data

Through IGS, read the temperature data, negative pressure value, cloth bag pressure difference, dust concentration, material level data and converter current value, voltage value and frequency value in PLC.

4) Data will be stored in real-time database

Create points in a real-time database through Proficy client, and store all relevant data in a real-time database for subsequent data processing.

5) IFIX display and alarm Settings

Create a corresponding point on the iFIX interface to read the data received by IGS and display it, and then configure the alarm Settings for that data.

6) Real-time push of risk warning information

When the data exceeds the set warning value during the operation of the device, the relevant person in charge will be notified via the company's 0A platform SMS, which includes the time, point and specific situation of the risk.

7) Establishment of equipment risk point database

After the risk point is processed, the processing situation of the risk point shall be explained in detail, and relevant contents such as processing method and effect 
after processing shall be entered into Oracle database. The next time a similar situation occurs, it will be automatically called so that the risk can be quickly identified and dealt with.

The establishment of the system completely breaks the shackles of traditional manual risk identification based on experience and the inability to quickly and effectively judge potential risks. Through the identification, control and early warning of operational system risks of power energy supply, the following objectives are realized in terms of functions: 1.Real-time data monitoring (second level) and historical data modeling analysis; 2.Multiplatform collaborative control; 3.Rapid and accurate prediction and early warning; 4 . Intelligent reference to the current situation of equipment and equipment maintenance plan prompt equipment maintenance; 5 . Monitor the instability factors in the production and process control. Finally, it can reduce the equipment failure rate, reduce the potential safety hazards, and keep the kinetic energy supply stable and efficient for a long time.

\section{Conclusion}

This paper first introduces in detail the research content of risk control system establishment: system architecture, system deployment risk point system, control data platform, data processing mode; Then the practical application and beneficial analysis of the system in the equipment of dust removal, moisture removal and odor removal in our factory are introduced. Since the equipment of tobacco industry has a high degree of similarity, the system has a high reference value in the practical application of our factory.

\section{References}

1. Guo Chaohao.Research on smart Grid Security Control based on intermittent renewable energy interconnection [D]. North China Electric Power University, North China Electric Power University(Beijing),2017.DOI: 10.7666/d.Y3264420.

2. Zheng Mingyu, Dong Zheng, $\mathrm{Hu}$ Yongsheng.Discussion on quality assurance method of Equipment Supervision based on risk Management [J] China Equipment Engineering,2020(10):53-54.

3. Huang Lijie, Brief Discussion on Environmental Safety Risk Management of Power Supply Equipment operation [J]. Science and Technology Innovation and Application,2017,(35):133-134.

4. wang huifang, Yang hejuan, he pentium, et al. Improvement analysis of state failure rate model of power transmission and transformation equipment [J] power system automation 2011,3(16:27-31,43).

5. Zhang Yige, Risk control business process analysis and optimization Strategy research of Central and Southern Air Traffic Control System [D]. South China University of Technology,2018.
6. Zhang Guangbiao, Discussion on the Establishment of silk Control alarm System in Tobacco Factory [J]. China Equipment Engineering,2015(09):56-57.

7. liao huan's application of IFIX in centralized monitoring system of tobacco factory [J]. Journal of hunan university of science and technology,2012,33(11):109-11.

8. Jie Daoliang, Research and Practice of Automatic Data Acquisition System for Tobacco Enterprises [J]. Automation Instrument,2006(03):52-55.

9. Chaoyang Fu'Ji ashen Zheng, Jingmao Zhao, et. al. Application of grey relational analysis for corrosion failure of oil tubes $[\mathrm{J}]$. Corrosion Science,2001,43(5):81-89.

10. Miguel Aria-Albomoz, Heman.Sanhueza Hardy. Distribution Networl Con69uration for Minimum Enemy Supply Cost[J].IEEE Transactions on Power Systems. 2004,19(1): 38-542.

11. Liu LAN, Research and Application of port production operation Risk Control Training and Examination System [D]. Tianjin: Tianjin University of Technology,2014, DOI: 10.7666/ d.Y2519605. 